\title{
Carrier testing in minors: a systematic review of guidelines and position papers
}

\author{
Pascal Borry ${ }^{*},{ }^{1}$ Jean-Pierre Fryns ${ }^{2}$, Paul Schotsmans ${ }^{1}$ and Kris Dierickx ${ }^{1}$ \\ ${ }^{1}$ Centre for Biomedical Ethics and Law, Catholic University of Leuven, Leuven, Belgium; ${ }^{2}$ Department of Human \\ Genetics, Catholic University of Leuven, Leuven, Belgium
}

The objective of this article is to review all published normative ethical and clinical guidelines concerning the genetic carrier testing of minors. The databases Medline, Philosopher's Index, Biological Abstracts, Web of Science, and Google Scholar were searched using keywords relating to the carrier testing of children. We also searched the websites of the national bioethics committees indexed on the websites of WHO and the German Reference Center for Ethics in the Life Sciences, the Human Genetics Societies of various nations indexed on the website of the International Federation of Human Genetics Societies and related links, and the national medical associations indexed on the website of the World Medical Association. We retrieved 14 guidelines emanating from 24 different groups. All guidelines advanced the following preferences: (1) carrier testing should not be performed in children, and (2) testing should be deferred until the child can give proper informed consent to be tested. The guidelines varied in three areas: (a) the role of genetic services in ensuring that children are informed about their carrier status and associated risks when they are older; (b) exceptions to the general rule of withholding or deferring carrier testing; and (c) the communication of incidentally discovered carrier status. In the absence of compelling reasons, carrier testing of a child can reasonably be deferred until the child has the intellectual capacity needed to discern if and when to be tested.

European Journal of Human Genetics (2006) 14, 133-138. doi:10.1038/sj.ejhg.5201509; published online 2 November 2005

Keywords: ethics; minors; carrier testing

\section{Introduction}

While a great deal of information has been published on the ethical aspects of predictive genetic testing of minors, much less has been written on the ethical aspects of carrier testing of children or adolescents. ${ }^{1}$ Carrier tests are performed to determine whether a person carries a mutated gene or balanced chromosomal rearrangement and are relevant for autosomal recessive and X-linked

*Correspondence: P Borry, Center for Biomedical Ethics and Law, KU Leuven, Kapucijnenvoer 35/3, Leuven, 3000, Belgium.

Tel: + 32163369 51; Fax: + 32163369 52;

E-mail: Pascal.Borry@med.kuleuven.be

No competing interests

Received 9 May 2005; revised 19 July 2005; accepted 16 September 2005; published online 2 November 2005 disorders as well as for chromosomal abnormalities. Various agencies have underscored the need for comprehensive and consistent policies that regulate genetic services, in general, ${ }^{2,3}$ and that normalise genetic testing of minors, in particular, and the need for all testing agents to apply these policies. The objective of this article is to promote the latter through a review of all published normative ethical and clinical guidelines on carrier testing of minors in families affected by autosomal recessive or $\mathrm{X}$-linked disorders or by balanced chromosomal rearrangements. Our focus is on cascade screening in which carrier testing is offered to those with a family history of such a disorder and not on general or unfocused population screening. We start with the assumption that it is desirable and possible for healthcare professionals to work towards a consensus on these issues. 


\section{Methods}

\section{Data sources}

We searched the databases Medline, Philosopher's Index, Biological Abstracts, Francis, Web of Science, Current Contents, and Google Scholar using the following search strategy: (Child OR childhood OR adolescen* OR infant OR young OR paediatric OR pediatric OR newborn OR minor) AND (genetic OR carrier) AND (ethic* OR guideline OR position OR bioethic* OR moral OR autonomy OR normative OR statement OR report OR recommendation). We also searched the websites of numerous national bioethics committees (listed on the websites of WHO and the German Reference Center for Ethics in the Life Sciences), the websites of the Human Genetics Societies of different nations (listed on the website of the International Federation of Human Genetics Societies and related links), and the websites of several national medical associations (listed on the website of the World Medical Association).

\section{Study selection}

Articles were eligible for inclusion in our review if they were position papers or reports, or if they contained guidelines or statements emanating from international and national organisations, bioethics committees, and professional associations that explicitly addressed carrier testing of children and/or adolescents in families affected by an autosomal recessive or X-linked disorder or by balanced chromosomal rearrangements. We focused our search on general, not disease-specific, statements and excluded guidelines that focused on genetic testing related to adoption. We included in our study only guidelines written in English or guidelines translated into English.

\section{Data extraction and synthesis}

In contrast to systematic reviews focusing on the relevant research involving the accuracy and precision of diagnostic tests; the power of prognostic markers; and the efficacy and safety of therapeutic, rehabilitative and preventive regimens, our systematic review of normative positions has two aims: (1) to assemble 'a reliable and comprehensive account of the facts of the matter and to identify and clarify concepts that are relevant to the valuation of the ethical implications of those facts', and (2) to organise 'these concepts into an argument (a coherent set of reasons that together support a conclusion for how one should or should not act)'. ${ }^{4}$ This systematic review of normative ethical positions towards carrier testing is based on a formal tool developed by McCullough. ${ }^{4}$

\section{Results}

\section{Recommendations from ethical guidelines}

In total, we retrieved 14 guidelines (Table 1) emanating from 24 different groups. The guidelines originated from genetic associations and societies (11), medical and paediatric associations and institutes (10), and government-related organisations (2). Only one originated from a consumer group. All the guidelines were in agreement that children preferably should not undergo carrier testing and that testing of children ideally should be deferred. All guidelines stated that it is in a child's best interest for him to decide whether to be tested at some stage later in life. The child's future autonomy tended to be the main ethical argument at stake. As carrier testing has the potential of affecting the future reproductive prospects of a child, the guidelines emphasised that the decision to test should be made by the child when he reaches reproductive age. This view is based on the basic ethical principle of informed consent, by which an individual can freely and voluntarily give, without external pressure, his consent to be tested after being informed of the benefits, risks, procedures, and other pertinent information relating to the carrier test. As carrier testing performed during childhood only affects the future of that child, not that of his parents or guardians, the guidelines stated that it is wiser to defer testing until the child himself is able to give proper informed consent than to acquiesce to the wishes of his parents or guardians to go forward with testing. The child's personal consent takes precedence over the wishes of third parties, including parents, either to carry out or to refuse genetic testing. Knowledge of carrier status critically impacts future decisions concerning reproduction (eg, carrier testing of partner, prenatal diagnoses, artificial insemination, preimplantation genetic diagnoses, adoption, not to have children). Some guidelines suggested that carrier testing performed during childhood also denies the child of confidentiality, a right he would expect if tested as an adult.

\section{Recommended age for carrier testing}

All guidelines agreed implicitly that the legal standard of 18 years of age represents the borderline between childhood and adulthood. While several guidelines made a distinction between childhood and adolescence, an exact range of ages was not attached to this distinction. The differences that distinguish children from adolescents refer to the gradual development of a child's cognitive skills and moral reasoning and the fact that 'as children progress through successive states of development, they become capable of greater participation in decisions about their own welfare'. 5 When adolescents meet 'conditions of competence, voluntariness, and adequate understanding of information', 5 are 'able to participate in the decision as an autonomous individual', 6 have 'decision-making capacity', $^{7}$ or are mature enough to take control of his or her own healthcare, they can be considered to be mature enough to request a carrier test. In this context, some have argued that further consultation with other genetic service providers, paediatricians, psychologists, and ethics 
Table 1 Overview of ethical and clinical guidelines and corresponding position statements for recommending carrier testing of minors within a family context

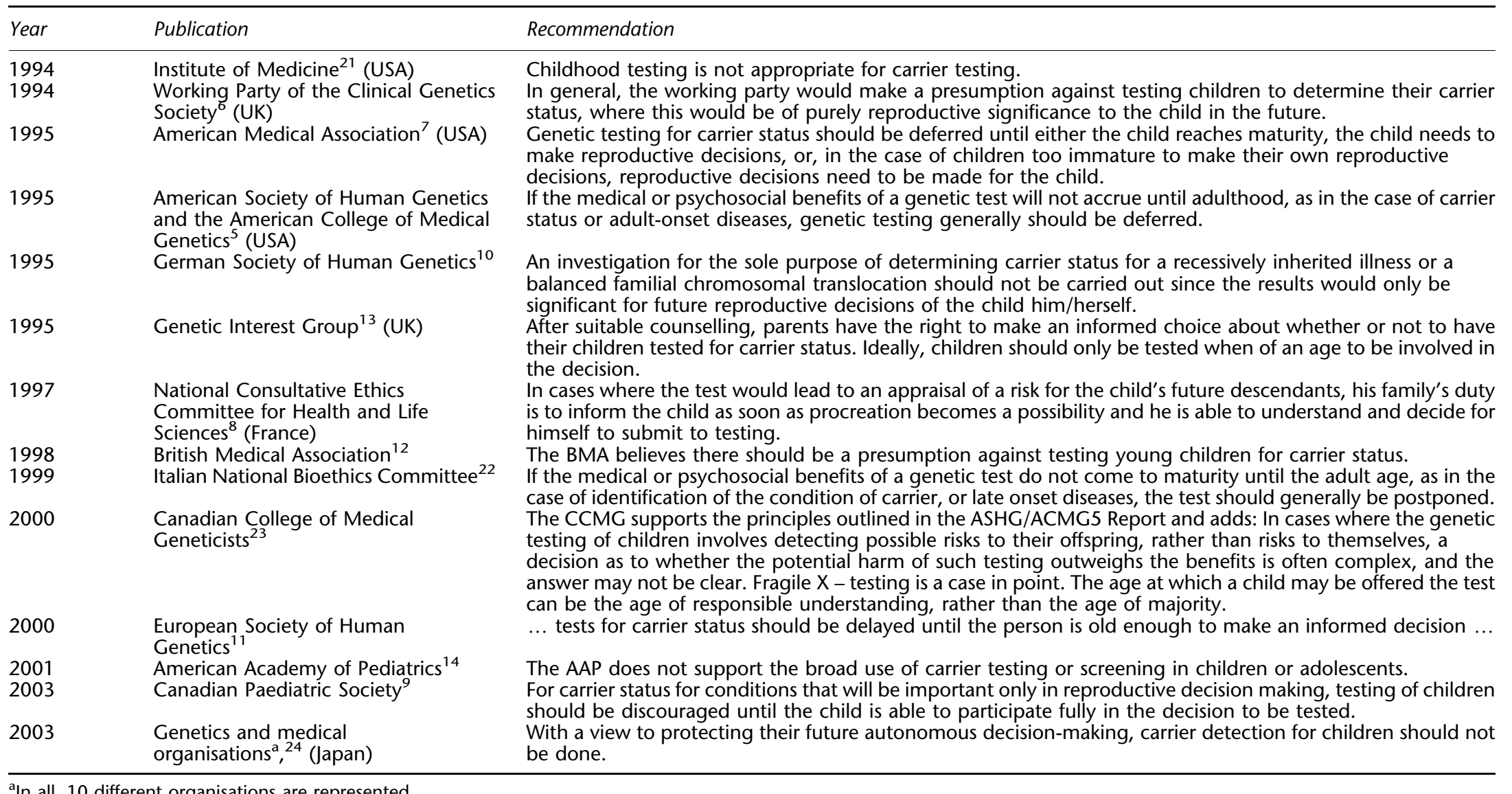

an all, 10 different organisations are represented. 
committees may be appropriate to evaluate whether the adolescent in question fully meets these criteria. ${ }^{5}$ Moreover, it is clearly advisable that the adolescent has his parents' support.

\section{Recommendations for informing the child}

While these guidelines focused on the ethical aspects of performing carrier testing in minors, they failed to address the issue of informing them of their genetic risk. It is likely that most of these guidelines assumed that parents, being inherently responsible for the welfare of their children, are also responsible for informing their child of his genetic risk. The guideline penned by the French National Consultative Ethics Committee for Health and Life Sciences confirms this assumption, by stating that it is the duty of parents to inform their child of any genetic risk when that child reaches reproductive age. ${ }^{8}$ Similarly, the guideline of the Canadian Paediatric Society encourages open discussion regarding familial genetic risk in an ageappropriate manner within the context of the family unit, ${ }^{9}$ and the guideline of the German Society of Human Genetics bestows the duty of informing children of genetic risks on parents. ${ }^{10}$ The latter limits the role of the genetic counsellor to educating parents about inherited disorders so that they clearly understand the significance of carrier testing and can responsibly inform their child about potential genetic risks at a later date. Only guidelines from the Working Party of the Clinical Genetics Society of the UK assigned a clear responsibility to genetic counsellors, stressing that it is the responsibility of both the family and the healthcare system to ensure that carrier testing is offered when the child is older. ${ }^{6}$

\section{Recommendations for exceptional cases}

Five guidelines ${ }^{6-8,10,11}$ addressed circumstances by which the carrier status of a child can assist the genetic counselling of family members. These guidelines stipulated that such cases should be considered carefully, and indicated that, in the majority of cases, pertinent genetic information can be obtained without testing the child, that is, carrier testing of parents can also provide this information and thus should take precedence over the testing of children. These guidelines endorsed carrier testing of children only if the test results will provide critical information to a relative that cannot be obtained by other means. Two of these guidelines elaborated further, stating that carrier testing of children should be done if the test results will medically benefit family members (eg, by providing haplotype information). ${ }^{7,11}$ In this regard, the test results must prevent substantial harm to a family member and there must not exist any reasonable alternative to avoid harm. ${ }^{8}$

Two guidelines, one published by the British Medical Association $^{12}$ (BMA) and the other by the UK-based Genetic Interest Group ${ }^{13}$ (GIG), presented a more flexible view of the testing of children. These two guidelines primarily focused on family dynamics and the individual concerns of family members, stressing that the interests of a child cannot be considered separately from those of his parents and other family members. These guidelines posited that, in the vast majority of cases, agreement to defer carrier testing will be reached after healthcare professionals and parents openly discuss the positive and negative consequences of testing. These guidelines also underscored that the obstinate refusal to comply with a parental request for the carrier testing of a child (eg, in cases where the parents cannot deal with the anxiety of not knowing the carrier status of their child) may have a more negative impact on the child and his family than would complying with the request. Both stated that having knowledge early on of one's carrier status could help a child to cope with this information starting in childhood and could reduce the anxiety and uncertainty experienced by parents about their child's carrier status. The two guidelines differed, however, in their interpretation of the term 'best interests'. The BMA guideline defined 'best interests' in a broad sense, incorporating psychological, social, familial, and medical factors to ascertain the most appropriate course of action. By contrast, the GIG guideline, defined 'best interests' in a narrow sense, arguing for the right of parents to have their children tested.

\section{Recommendations for incidental discovery of carrier status}

Four guidelines discussed the course of action in cases in which carrier status was discovered incidentally (eg, during diagnostic testing, screening, or prenatal diagnosis, or in a research context). ${ }^{7,9,10,12}$ While guidelines from the $\mathrm{BMA}^{12}$ and the American Academy of Pediatrics ${ }^{14}$ recommended that carrier status results obtained incidentally should be conveyed to parents, the American Medical Association ${ }^{7}$ and the German Society of Human Genetics ${ }^{10}$ recommended that this information should not be disclosed to parents or to other third parties. Rather, this information should be discussed with the child when he reaches reproductive age. The guidelines from the American Medical Association also provided instructions for maintaining the confidentiality of this genetic information, stating that this privileged information should be kept in a separate portion of a patient's medical record to prevent accidental disclosure. $^{7}$

\section{Discussion}

Several international organisations have emphasised the need to treat genetic testing with special care by drafting guidelines that outline rules to ensure respect for human dignity and to protect privacy, that make provision for pretest education and psychological counselling, and that require obtaining meaningful informed consent before 
genetic testing is performed. ${ }^{15,16}$ Decisions regarding the testing of minors should be dealt with cautiously, taking into account the minors' best interests. In addition, if possible the opinion of the minors should be taken into consideration as a determining factor of whether testing should be performed. Of course, the weight of a minor's opinion is proportionate to his age and degree of maturity. These basic principles frame the concrete recommendation that carrier testing of children should be deferred until a child is capable of deciding whether to be tested. ${ }^{17}$

Although the great majority of ethical guidelines overseeing the carrier testing of children agree that testing should be deferred until the child is capable of making an informed decision of whether to be tested, two guidelines those penned by the BMA and GIG - expressed concerns about the rigid interpretation of this general rule, proposing instead for flexibility when exceptional situations are encountered. An important distinction between the BMA and GIG guidelines and the other guidelines lies in how much weight each puts on the psychological and social impact of carrier testing on the children themselves. Despite the lack of conclusive evidence that carrier testing performed during childhood harms children psychologically, $^{18}$ the great majority of genetic testing guidelines espouse the premise that carrier testing might be detrimental to the mental well being of tested children, and as such, should be disallowed in children. Both the BMA ${ }^{19}$ and $\mathrm{GIG},{ }^{13}$ however, acknowledge that available evidence (that testing is neither beneficial nor detrimental) is insufficient to warrant the global prohibition of carrier testing of children.

Although acknowledging that empirical research can have an important role in developing policy guidelines and in affecting ethical decision-making processes, ${ }^{20}$ we stress that the results from empirical research alone cannot determine what is good or bad, right, or wrong. The absence of data that carrier testing during childhood is not harmful would be a poor reason to use to promote the carrier testing of minors. Ethically salient issues, such as maintaining the child's right for autonomy, confidentiality, and privacy, must be primarily considered when developing formal guidelines for the carrier testing of children. These rights would be violated if a child was tested for no compelling reason and his carrier status information was relayed to his parents. Choices and actions involving sex and reproduction are part of the most personal and intimate aspects of life. As the ultimate purpose of carrier testing is to produce information that can guide future reproductive decisions, it is important that children, as they grow to adulthood, are given the opportunity to request testing and to have full control over their test information, sharing it with whom they desire. This viewpoint, however, should not restrain parents from openly discussing opportunities for carrier testing later in their children's life. Nonetheless, from a young age children should be advised of the risks associated with knowing one's carrier status, even though the actual testing is postponed. Next to the responsibility of parents, genetic service providers should follow up the genetic risk of a family. It is important to avoid situations by which minors are never informed about their genetic risk. In addition, genetic counselling should be made available to minors throughout the course of their lives, with the aim of helping them envisage undergoing carrier testing.

\section{Limitations}

Although we retrieved 14 different guidelines or statements that discussed the carrier testing of minors, only one statement was penned by a consumer group. This apparent lack of representation from patient organisations may be because we focused our search on general guidelines rather than on disease-oriented guidelines. Nevertheless, even after contacting various geneticists and important groups of patients organisations as the Genetic Interest Group (UK) and the Dutch Genetic Alliance (VSOP) we did not succeed in identifying any policy document on childhood genetic testing from disease-specific family support groups. Future research, for example surveying patient orgnisations on that issue could help identify if these groups have developed such policies, that maybe are not available online or not widely diffused.

\section{Conclusion}

As carrier testing has in most cases only implications for the reproductive health of the child, all guidelines recommend delaying carrier testing until the minors can participate themselves in these decisions that are affecting them. Several guidelines state that minors could request a carrier test from the moment they meet conditions of competence and have decision-making capacity. Most guidelines failed to address the issue of informing children of their genetic risk. The BMA and GIG presented a dissenting voice on carrier testing by stating that the obstinate refusal to comply with a parental request for the carrier testing of a child may have a more negative impact on the child and his family than would complying with this request. Further discussion on carrier testing in minors may be useful and a guideline on genetic testing in minors from European geneticists is recommended.

\section{Acknowledgements}

The research for this contribution was supported by the Eurogentest Network of Excellence of the EU, FP6-512148.

\section{References}

1 Davis DS: Discovery of children's carrier status for recessive genetic disease: some ethical issues. Genet Test 1998; 2: 323-327. 
2 Genetic services in Europe: a comparative study of 31 countries by the concerted action on genetic services in Europe. Eur J Hum Genet 1997; 5 (Suppl 2): 1-220.

3 Kofman S: Community genetic services in Latin America and regional networks on medical genetics. WHO: Geneva, 2003.

4 McCullough LB, Coverdale JH, Chervenak FA: Argument-based medical ethics: a formal tool for critically appraising the normative medical ethics literature. Am J Obstet Gynecol 2004; 191: 1097 - 1102 .

5 The American Society of Human Genetics Board of Directors and the American College of Medical Genetics Board of Directors: Points to consider: ethical, legal, and psychological impications of genetic testing in children and adolescents. Am J Hum Gen 1995; 57: $1233-1241$.

6 Clarke A: The genetic testing of children. Working party of the clinical genetics society (UK). J Med Genet 1994; 31: 785-797.

7 American Medical Association: Testing children for genetic status www.ama-assn.org, 1995.

8 National Consultative Ethics Committee for Health and Life Sciences: Opinion and recommendations on 'Genetics and medicine: from prediction to prevention'. Reports www.ccneethique.fr, 1995.

9 Bioethics Committee Canadian Paediatric Society: Guidelines for genetic testing of healthy children. Paediatr Child Health 2003; 8: $42-45$.

10 Committee for Public Relations and Ethical Issues of the German Society of Human Genetics: Statement on genetic diagnosis in children and adolescents www.gfhev.de, 1995.

11 European Society of Human Genetics: Provision of genetic services in Europe - current practices and issues www.eshg.org, 2001.

12 British Medical Association: Human genetics: choice and responsibility. Oxford University Press: Oxford, 1998.
13 Dalby S: GIG response to the UK clinical genetics society report 'The genetic testing of children'. J Med Genet 1995; 32: 490-491.

14 Committee on Bioethics: Ethical issues with genetic testing in pediatrics. Pediatrics 2001; 107: 1451-1455.

15 Council of Europe: Convention for the Protection of Human Rights and Dignity of the Human Being with regard to the application of Biology and Medicine: Convention on Human Rights and Biomedicine. Oviedo: Council of Europe, 1997.

16 UNESCO International Bioethics Committee: Report on genetic screening and testing. UNESCO: Paris, 1994.

17 Davis DS: Genetic dilemmas and the child's right to an open future. Hastings Cent Rep 1997; 27: 7-15.

18 Jarvinen $\mathrm{O}$, Hietala $\mathrm{M}$, Aalto $\mathrm{A}$, et al: A retrospective study of long-term psychosocial consequences and satisfaction after carrier testing in childhood in an autosomal recessive disease: aspartylglucosaminuria. Clin Genet 2000; 58: $447-454$.

19 British Medical Association: Medical Ethics Today. The BMA's handbook of ethics and law. London: BMA, 2004.

20 Borry P, Schotsmans P, Dierickx K: What is the contribution of empirical research in bioethics? An ethical analysis. Med Health Care Philos 2004; 7: 41-53.

21 Committee on Assessing Genetic Risk: Institute of Medicine: Assessing Genetic Risks Implications for Health and Social Policy. National Academy Press: Washington DC, 1994.

22 Italian Government National Bioethics Committee: Bioethical guidelines for genetic testing. Governo Italiano: Roma, 1999.

23 Canadian College of Medical Geneticists: Genetic testing of children www.ccmg.medical.org, 2005.

24 The Japan Society of Human Genetics: Council committee of ethics: guidelines for genetic testing. I Hum Genet 2001; 46: $163-165$. 